\title{
Causality and Loop-Tree Duality at Higher Loops
}

\author{
Robert Runkel, Zoltán Szőr, Juan Pablo Vesga, and Stefan Weinzierl \\ PRISMA Cluster of Excellence, Institut für Physik, Johannes Gutenberg-Universität Mainz, D-55099 Mainz, Germany
}

\author{
(Received 7 February 2019; published 22 March 2019)
}

\begin{abstract}
We relate an $l$-loop Feynman integral to a sum of phase space integrals, where the integrands are determined by the spanning trees of the original $l$-loop graph. Causality requires that the propagators of the trees have a modified $i \delta$ prescription, and we present a simple formula for the correct $i \delta$ prescription.
\end{abstract}

DOI: 10.1103/PhysRevLett.122.111603

Introduction.-Relating loop integrals to trees goes back to Feynman [1]. The Feynman tree theorem allows us to relate an $l$-loop Feynman integral with $N$ internal propagators to an $l$-fold phase space integral with a number of cuts $N_{\text {cut }}$ on the original integrand, with $N_{\text {cut }}$ ranging from $l$ to $N$. One could argue that a better name for this theorem would be the Feynman forest theorem, as in general (i.e., for $N_{\text {cut }}>l$ ) the integrand corresponds to a set of trees, i.e., a forest. This is not very convenient: If more than $l$ cuts are present, each additional cut imposes a nontrivial constraint on the phase space integration.

What we would like to have is a formula which relates an $l$-loop Feynman integral to an $l$-fold phase space integral without any additional constraints. The integrand of the phase space integral then corresponds to a tree, not a forest, and is obtained from the original integrand by exactly $l$ cuts. For one-loop integrals this was achieved in Ref. [2]. An important result of Ref. [2] was the statement that the uncut propagators have a modified $i \delta$ prescription. The usual $i \delta$ prescription for a Feynman propagator is

$$
\frac{i}{k_{j}^{2}-m_{j}^{2}+i \delta}
$$

where $\delta>0$ is an infinitesimal small quantity. The modified $i \delta$ prescription is a consequence of causality. We call propagators with a modified $i \delta$ prescription dual propagators.

In this Letter we present the generalization to an arbitrary loop number $l$. A comment is in order: A generalization of loop-tree duality to two loops and beyond has already been considered in Ref. [3]. However, the final formulas presented there are not particularly elegant and involve a mixture of Feynman propagators and one-loop dual

Published by the American Physical Society under the terms of the Creative Commons Attribution 4.0 International license. Further distribution of this work must maintain attribution to the author(s) and the published article's title, journal citation, and DOI. Funded by SCOAP ${ }^{3}$. propagators. Our result is more aesthetic: All uncut propagators are dual propagators, with a simple dual $i \delta$ prescription, which reduces in the one-loop case to the one of Catani et al. [2]. A dual propagator is of the form

$$
\frac{i}{k_{j}^{2}-m_{j}^{2}+i s_{j}(\sigma) \delta} \text {. }
$$

Only the sign of the function $s_{j}(\sigma)$ is relevant. The function $s_{j}(\sigma)$ depends on the energy $E_{j}$ and the energies of the cut propagators $E_{\sigma_{1}}, \ldots, E_{\sigma_{l}}$ and will be given in Eq. (27) or alternatively in Eq. (32) below.

Let us also briefly comment on the difference of the loop-tree duality approach with the so-called $Q$-cut approach [4]: The latter involves propagators linear in the loop momenta, where the information due to the infinitesimal imaginary part is lost if all propagators have the same small imaginary part as in Eq. (1). The i $\delta$ prescription is restored by introducing different infinitesimal small imaginary parts for the internal propagators and averaging over all possible relative orderings. In our approach, only propagators quadratic in the loop momenta occur. Furthermore, the modified $i \delta$ prescription of the dual propagators follows directly from the $i \delta$ prescription of the Feynman propagators. Our approach applies to massless and massive particles.

Notation.-Let $\Gamma$ be a Feynman graph with $l$ loops, $n$ external lines, and $N$ internal edges. We denote by $E_{\Gamma}=$ $\left\{e_{1}, \ldots, e_{N}\right\}$ the set of internal edges. A spanning tree for the graph $\Gamma$ is a subgraph $T$ of $\Gamma$, which contains all the vertices of $\Gamma$ and is a connected tree graph [5]. If $T$ is a spanning tree for $\Gamma$, then it can be obtained from $\Gamma$ by deleting $l$ internal edges, say, $\left\{e_{\sigma_{1}}, \ldots, e_{\sigma_{l}}\right\}$. We denote by $\sigma=\left\{\sigma_{1}, \ldots, \sigma_{l}\right\}$ the set of indices of the deleted edges and by $\mathcal{C}_{\Gamma}$ the set of all such sets. Thus, $\left|\mathcal{C}_{\Gamma}\right|$ gives the number of spanning trees for the graph $\Gamma$.

Each $\sigma \in \mathcal{C}_{\Gamma}$ also defines a cut graph $T_{\text {cut }}$, obtained by cutting each of the $l$ internal edges $e_{\sigma_{j}}$ into two half-edges. The $2 l$ half-edges become external lines of $T_{\text {cut }}$. The graph $T_{\text {cut }}$ is a tree graph with $n+2 l$ external lines. 
We denote the external momenta of the graph $\Gamma$ by $p_{1}, \ldots, p_{n}$ and the internal momenta by $k_{1}, \ldots, k_{N}$. We will assume that the internal momenta have been labeled such that the first $l$ internal momenta $k_{1}, \ldots, k_{l}$ form a basis of independent loop momenta. For each internal edge we set

$$
D_{j}=k_{j}^{2}-m_{j}^{2}+i \delta, \quad e_{j} \in E_{\Gamma} .
$$

We will assume that $D_{i} \neq D_{j}$ for $i \neq j$. Otherwise, we consider a reduced graph $\Gamma^{\prime}$ with edge $e_{j}$ contracted and a higher power of the propagator associated to edge $e_{i}$.

For a function $f$ depending on a $D$-dimensional momentum variable $k=(E, \vec{k})$, where the vector $\vec{k}$ is $(D-1)$ dimensional, we write either $f(k)$ or $f(E, \vec{k})$. We would like to integrate the function $f$ over the hyperboloid $k^{2}=m^{2}$. The quantities

$$
\int \frac{d^{D-1} k}{(2 \pi)^{D-1} 2 \sqrt{\vec{k}^{2}+m^{2}}} f\left( \pm \sqrt{\vec{k}^{2}+m^{2}}, \vec{k}\right)
$$

give the integrals over the forward hyperboloid and the backward hyperboloid, respectively. As a shorthand notation, we set

$$
\begin{aligned}
& \int \frac{d^{D-1} k}{(2 \pi)^{D-1} 2 \sqrt{\vec{k}^{2}+m^{2}}} f(k) \\
& =\int \frac{d^{D-1} k}{(2 \pi)^{D-1} 2 \sqrt{\vec{k}^{2}+m^{2}}} \\
& \quad \times\left[f\left(\sqrt{\vec{k}^{2}+m^{2}}, \vec{k}\right)+f\left(-\sqrt{\vec{k}^{2}+m^{2}}, \vec{k}\right)\right]
\end{aligned}
$$

for the integral over the forward and the backward hyperboloid.

Loop-tree duality.-Let $P_{\Gamma}$ be a polynomial in the loop momenta. We consider

$$
I=\int\left(\prod_{j=1}^{l} \frac{d^{D} k_{j}}{(2 \pi)^{D}}\right) \frac{P_{\Gamma}}{\prod_{e_{j} \in E_{\Gamma}} D_{j}^{\nu_{j}^{j}}} .
$$

We split each loop integration into an integration over the energy and the spatial components of the loop momentum:

$I=\int\left(\prod_{j=1}^{l} \frac{d^{D-1} k_{j}}{(2 \pi)^{D-1}}\right) \frac{1}{(2 \pi)^{l}} \int \frac{P_{\Gamma} d E_{1} \wedge \cdots \wedge d E_{l}}{\prod_{e_{j} \in E_{\Gamma}} D_{j}^{\nu_{j}}}$.

We perform the energy integrations with the help of the residue theorem. Let us assume that the polynomial $P_{\Gamma}$ is such that all energy integrations over half-circles at infinity vanish. This assumption is always satisfied for scalar integrals where $P_{\Gamma}=1$. If this assumption is not met, we may enforce it by subtracting local ultraviolet counterterms from the integrand [6,7].
Let $\mathcal{E} \subset \mathbb{C}^{l}$ be the set of points $E=\left(E_{1}, \ldots, E_{l}\right)$, where $l$ internal propagators go on shell and removing the corresponding edges gives a spanning tree. We have

$$
|\mathcal{E}|=2^{l}\left|\mathcal{C}_{\Gamma}\right|
$$

This number is easily obtained from the number of spanning trees and the $2^{l}$ solutions per spanning tree. For generic values of $p_{1}, \ldots, p_{n}$ and $\vec{k}_{1}, \ldots, \vec{k}_{l}$ the points of $\mathcal{E}$ are distinct. Points in $\mathcal{E}$ coincide if in addition to the cut propagators one or more uncut propagators go on shell. We distinguish the cases of a pinch singularity and a nonpinch singularity. For a nonpinch singularity we may deform the integration contour for the spatial variables $\vec{k}_{1}, \ldots, \vec{k}_{l}$ into the complex domain. The modified $i \delta$ prescription given in Eq. (27) tells us in which direction we should deform. This is exactly the purpose of the present Letter. For a pinch singularity we have an infrared singularity. This singularity is either regulated by dimensional regularization or canceled in the combination with real contributions according to the Kinoshita-Lee-Nauenberg theorem $[8,9]$.

Let $\sigma \in \mathcal{C}_{\Gamma}$ be a set of indices defining a spanning tree. For each cut edge we choose an orientation and we may take the $l$ independent loop momenta to be the loop momenta flowing through the edges $e_{\sigma_{1}}, \ldots, e_{\sigma_{l}}$ with the chosen orientation. Let

$$
E_{\sigma}^{(\alpha)}=\left(E_{\sigma_{1}}^{(\alpha)}, \ldots, E_{\sigma_{l}}^{(\alpha)}\right)
$$

be a solution to

$$
D_{\sigma_{1}}=\cdots=D_{\sigma_{l}}=0
$$

In total there are $2^{l}$ solutions, $E_{\sigma}^{(1)}, \ldots, E_{\sigma}^{\left(2^{l}\right)}$, given by

$$
\left( \pm \sqrt{\vec{k}_{\sigma_{1}}^{2}+m_{\sigma_{1}}^{2}-i \delta}, \ldots, \pm \sqrt{\vec{k}_{\sigma_{l}}^{2}+m_{\sigma_{l}}^{2}-i \delta}\right) .
$$

Let us denote by $n_{\sigma}^{(\alpha)}$ the number of times the negative root $-\sqrt{\cdots}$ occurs in $E_{\sigma}^{(\alpha)}$. We set

$$
f=\frac{P_{\Gamma}}{\prod_{e_{j} \in E_{\Gamma}} D_{j}^{\nu_{j}}} .
$$

We define the local residue [10] at $E_{\sigma}^{(\alpha)}$ by

$$
\operatorname{res}\left(f, E_{\sigma}^{(\alpha)}\right)=\frac{1}{(2 \pi i)^{l}} \oint_{\gamma_{\varepsilon}} f d E_{1} \wedge \cdots \wedge d E_{l}
$$

The integration in Eq. (13) is around a small $l$-torus,

$$
\gamma_{\varepsilon}=\left\{\left(E_{1}, \ldots, E_{l}\right) \in \mathbb{C}^{l}|| D_{\sigma_{i}} \mid=\varepsilon\right\},
$$


encircling $E_{\sigma}^{(\alpha)}$ with orientation

$$
d \arg D_{\sigma_{1}} \wedge d \arg D_{\sigma_{2}} \wedge \cdots \wedge d \arg D_{\sigma_{l}} \geq 0 .
$$

We consider

$$
\sum_{\alpha=1}^{2^{l}}(-1)^{n_{\sigma}^{(\alpha)}} \operatorname{res}\left(f, E_{\sigma}^{(\alpha)}\right)
$$

Let us make one remark: Equation (16) is not a global residue for the ideal $\left\langle D_{\sigma_{1}}^{\nu_{\sigma_{1}}}, \ldots, D_{\sigma_{l}}^{\nu_{\sigma_{l}}}\right\rangle$, due to the additional factor $(-1)^{n_{\sigma}^{(\alpha)}}$. The standard definition of the global residue for $\left\langle D_{\sigma_{1}}^{\nu_{\sigma_{1}}}, \ldots, D_{\sigma_{l}}^{\nu_{\sigma_{l}}}\right\rangle$ is just the sum over the $2^{l}$ local residues, without any sign factors. This sum vanishes, whereas the sum in Eq. (16) in general does not.

Proposition 1.-The expression in Eq. (16) is independent of the orientation of the edges $e_{\sigma_{1}}, \ldots, e_{\sigma_{l}}$.

Proof.-It is sufficient to show this for the case where we change the orientation of a single edge $e_{\sigma_{a}}$. We may use partial fraction decomposition in the variable $E_{\sigma_{a}}$. It is sufficient to show this for the case

$$
f\left(E_{\sigma_{a}}\right)=\frac{r_{+}}{E_{\sigma_{a}}-c}+\frac{r_{-}}{E_{\sigma_{a}}+c},
$$

where $r_{+}, r_{-}$and $c$ are $E_{\sigma_{a}}$ independent, since higher poles and polynomial terms do not contribute to the residue. (Polynomial terms are absent due to our assumption that the integration over half-circles at infinity vanishes.) We have

$$
\sum_{\alpha=1}^{2}(-1)^{n^{(\alpha)}} \operatorname{res}\left(f, E_{\sigma_{a}}^{(\alpha)}\right)=r_{+}-r_{-} .
$$

Now let us change the orientation. We set $E_{\sigma_{a}}^{\prime}=-E_{\sigma_{a}}$ and

$$
f^{\prime}\left(E_{\sigma_{a}}^{\prime}\right)=f\left(E_{\sigma_{a}}\right)=-\frac{r_{+}}{E_{\sigma_{a}}^{\prime}+c}-\frac{r_{-}}{E_{\sigma_{a}}^{\prime}-c} .
$$

Then

$$
\sum_{\alpha=1}^{2}(-1)^{n^{\prime(\alpha)}} \operatorname{res}\left(f^{\prime}, E^{\prime} \sigma_{a}^{(\alpha)}\right)=-r_{-}+r_{+} .
$$

Theorem 1.-With $f$ as in Eq. (12), we have

$$
\begin{gathered}
\frac{1}{(2 \pi)^{l}} \int f d E_{1} \wedge \cdots \wedge d E_{l}= \\
\frac{(-i)^{l}}{2^{l}} \sum_{\sigma \in \mathcal{C}_{\Gamma}} \sum_{\alpha=1}^{2^{l}}(-1)^{n_{\sigma}^{(\alpha)}} \operatorname{res}\left(f, E_{\sigma}^{(\alpha)}\right),
\end{gathered}
$$

where the contour of integration on the left-hand side is along the real axes separating the poles at $+\sqrt{\cdots}$ from the poles at $-\sqrt{\cdots}$.

Proof.-We prove Eq. (21) by induction on the number of loops $l$. We start with $l=1$. The function $f$ is given in this case by

$$
f=\frac{P_{\Gamma}}{\prod_{j=1}^{N} D_{j}^{\nu_{j}}} .
$$

Closing the contour below sums up the residues in the lower complex half-plane and we obtain

$$
\frac{1}{2 \pi} \int f d E=-i \sum_{j=1}^{N} \operatorname{res}\left(f, \sqrt{\vec{k}_{j}^{2}+m_{j}^{2}-i \delta}\right) .
$$

The minus sign comes from the clockwise orientation of the contour. If, on the other hand, we close the contour above, we pick up the residues in the upper complex half-plane and we obtain

$$
\frac{1}{2 \pi} \int f d E=i \sum_{j=1}^{N} \operatorname{res}\left(f,-\sqrt{\vec{k}_{j}^{2}+m_{j}^{2}-i \delta}\right) .
$$

Both Eqs. (23) and (24) express the original integral as a sum of residues, and so does the average of the two:

$$
\begin{aligned}
\frac{1}{2 \pi} \int f d E= & \frac{(-i)}{2} \sum_{j=1}^{N}\left[\operatorname{res}\left(f, \sqrt{\vec{k}_{j}^{2}+m_{j}^{2}-i \delta}\right)\right. \\
& \left.-\operatorname{res}\left(f,-\sqrt{\vec{k}_{j}^{2}+m_{j}^{2}-i \delta}\right)\right] .
\end{aligned}
$$

This agrees with Eq. (21) for the case $l=1$.

Let us now assume that Eq. (21) is correct for graphs with $(l-1)$ loops. We show that Eq. (21) holds for graphs with $l$ loops. Consider a graph $\Gamma$ with $l$ loops and pick a cycle (i.e., a closed path) in this graph. To this cycle corresponds an energy integration, and we may take the residues as we did in the one-loop case and average over the two cases obtained by closing the contour in the upper (lower) complex half-plane. Taking a single residue will put one edge of the cycle on shell and will fix the energy of this edge. This corresponds to cutting the cycle at this edge, and we obtain a graph $\Gamma^{\prime}$ with $(l-1)$ loops. For $\Gamma^{\prime}$ we may use the induction hypothesis, and doing this for every single residue gives Eq. (21).

Let us now specialize to the case $\nu_{1}=\cdots=\nu_{N}=1$ and let us work out the $i \delta$ prescription for the uncut propagators.

Theorem 2.- Let us assume again that $P_{\Gamma}$ is a polynomial in the loop momenta such that all energy integrations over half-cycles at infinity vanish. If all propagators occur to power one, we have 


$$
\begin{aligned}
\int & \left(\prod_{j=1}^{l} \frac{d^{D} k_{j}}{(2 \pi)^{D}}\right) \frac{P_{\Gamma}}{\prod_{e_{j} \in E_{\Gamma}}\left(k_{j}^{2}-m_{j}^{2}+i \delta\right)} \\
= & \frac{(-i)^{l}}{2^{l}} \sum_{\sigma \in \mathcal{C}_{\Gamma}} \int\left(\prod_{j=1}^{l} \frac{d^{D-1} k_{\sigma_{j}}}{(2 \pi)^{D-1} 2 \sqrt{\vec{k}_{\sigma_{j}}^{2}+m_{\sigma_{j}}^{2}}}\right) \\
& \times \frac{P_{\Gamma}}{\prod_{j \notin \sigma}\left[k_{j}^{2}-m_{j}^{2}+i s_{j}(\sigma) \delta\right]},
\end{aligned}
$$

where $s_{j}(\sigma)$ is defined as

$$
s_{j}(\sigma)=\frac{E_{j}}{E_{\|}}
$$

and $E_{\|}$is defined as follows: The set $\sigma=\left\{\sigma_{1}, \ldots, \sigma_{l}\right\} \in \mathcal{C}_{\Gamma}$ defines a tree $T_{\text {cut }}$ obtained from the graph $\Gamma$ by cutting the internal edges $C_{\sigma}=\left\{e_{\sigma_{1}}, \ldots, e_{\sigma_{l}}\right\}$. Cutting in addition the edge $e_{j} \in E_{\Gamma} \backslash C_{\sigma}$ will give a two-forest $\left(T_{1}, T_{2}\right)$. We orient the external momenta of $T_{1}$ such that all momenta are outgoing. Let $\pi$ be the set of indices corresponding to the external edges of $T_{1}$ which come from cutting the edges $C_{\sigma}$ of the graph $\Gamma$. The set $\pi$ may contain an index twice; this is the case if both half-edges of a cut edge belong to $T_{1}$. Then define $E_{\|}$by

$$
\frac{1}{E_{\|}}=\sum_{a \in\{j\} \cup \pi} \frac{1}{E_{a}}
$$

Proof.-Theorem 2 is a specialization of Theorem 1 to the case where the integrand has only single poles. The calculation of the residues yields

$$
\begin{array}{r}
\operatorname{res}\left(\frac{1}{D_{\sigma_{j}}}, \sqrt{\vec{k}_{\sigma_{j}}^{2}+m_{\sigma_{j}}^{2}-i \delta}\right)=\frac{1}{2 \sqrt{\vec{k}_{\sigma_{j}}^{2}+m_{\sigma_{j}}^{2}}}, \\
-\operatorname{res}\left(\frac{1}{D_{\sigma_{j}}},-\sqrt{\vec{k}_{\sigma_{j}}^{2}+m_{\sigma_{j}}^{2}-i \delta}\right)=\frac{1}{2 \sqrt{\vec{k}_{\sigma_{j}}^{2}+m_{\sigma_{j}}^{2}}},
\end{array}
$$

where we neglected on the right-hand side the infinitesimal small imaginary part. It remains to work out the sign of the imaginary part of the uncut propagators. Let us consider $D_{j}$ and with the notation as above the tree $T_{1}$. The external edges of $T_{1}$ are given by $e_{j}$, the set $E_{\mathrm{cut}}=\left\{e_{\pi_{1}}, e_{\pi_{2}}, \ldots\right\}$, and possibly a subset $E_{\text {ext }}$ of the external edges $\{1, \ldots, n\}$ of the original graph $\Gamma$. Energy conservation relates $E_{j}$ to minus the sum of the energies of all other external particles of the tree $T_{1}$. The energies corresponding to the edges from $E_{\text {ext }}$ are real; the energies corresponding to the edges from $E_{\text {cut }}$ have an infinitesimal small imaginary part. Taylor expansion to first order in $\delta$ gives

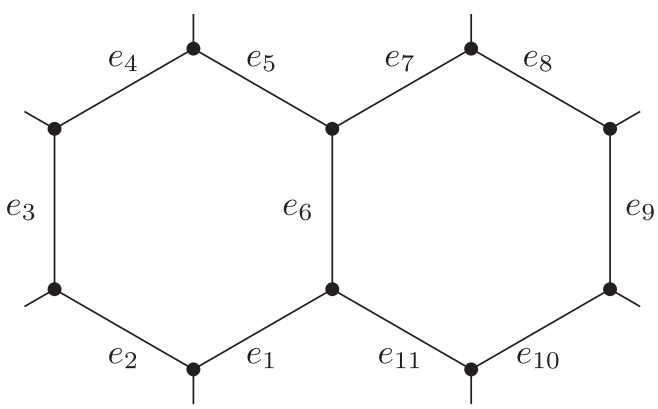

FIG. 1. A two-loop eight-point function with 11 propagators.

$$
\begin{aligned}
E_{\pi_{a}} & = \pm \sqrt{\vec{k}_{\pi_{a}}^{2}+m_{\pi_{a}}^{2}-i \delta} \\
& = \pm \sqrt{\vec{k}_{\pi_{a}}^{2}+m_{\pi_{a}}^{2}} \mp \frac{1}{2} \frac{i \delta}{\sqrt{\vec{k}_{\pi_{a}}^{2}+m_{\pi_{a}}^{2}}}+\mathcal{O}\left(\delta^{2}\right) .
\end{aligned}
$$

By a slight abuse of notation, we denote the $\mathcal{O}\left(\delta^{0}\right)$ term again by $E_{\pi_{a}}$. Thus, the replacement

$$
E_{\pi_{a}} \rightarrow E_{\pi_{a}}-\frac{i \delta}{2 E_{\pi_{a}}}
$$

makes the infinitesimal imaginary part explicit. Let us now look at $D_{j}$ and expand to first order in $\delta$ :

$$
\begin{aligned}
D_{j} & =k_{j}^{2}-m_{j}^{2}+i \delta=E_{j}^{2}-\vec{k}_{j}^{2}-m_{j}^{2}+i \delta \\
& =\left(\sum_{a \in \pi} E_{a}+\sum_{a \in E_{\mathrm{ext}}} E_{a}^{\mathrm{ext}}\right)^{2}-\vec{k}_{j}^{2}-m_{j}^{2}+i \delta \\
& \rightarrow k_{j}^{2}-m_{j}^{2}+\left(1+E_{j} \sum_{a \in \pi} \frac{1}{E_{a}}\right) i \delta+\mathcal{O}\left(\delta^{2}\right) .
\end{aligned}
$$

For the $\mathcal{O}(\delta)$ term, we have

$$
1+E_{j} \sum_{a \in \pi} \frac{1}{E_{a}}=E_{j}\left(\sum_{a \in\{j\} \cup \pi} \frac{1}{E_{a}}\right)=\frac{E_{j}}{E_{\|}} .
$$

Although we singled out the tree $T_{1}$ from the two-forest $\left(T_{1}, T_{2}\right)$, it is easily checked that the definition of $s_{j}(\sigma)$ is invariant under the exchange $T_{1} \leftrightarrow T_{2}$.

Theorem 2 is the main result of this Letter. It allows us to express a Feynman integral with no raised propagators as a sum of phase space integrals. Each phase space integral corresponds to a spanning tree of the original graph. The integrand of each phase space integral corresponds to a cut graph, where exactly $l$ internal propagators have been cut and the remaining $(\mathrm{N}-l)$ internal propagators have a modified $i \delta$ prescription given by Eq. (27). Theorem 2 is the specialization of Theorem 1 to Feynman integrals with no raised propagators. For Feynman integrals with raised propagators, we may still use Theorem 1 . The only change is that the computation of the residues is more involved. 


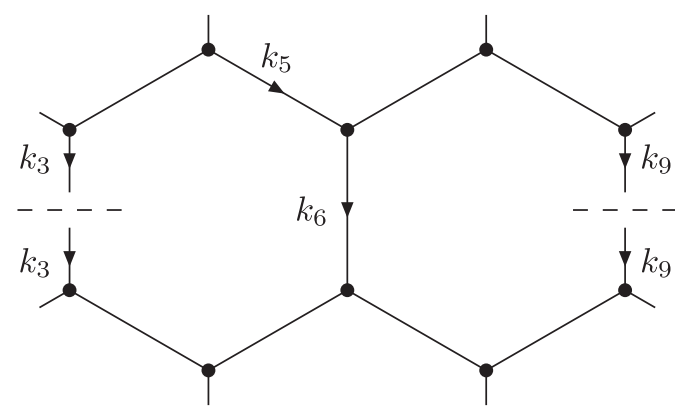

FIG. 2. A cut diagram corresponding to $\sigma=\{3,9\}$. We also indicate an orientation for the edges $e_{3}, e_{5}, e_{6}$, and $e_{9}$.

Residues of Feynman integrands with raised propagators have been considered in Ref. [11].

Although we defined the modified $i \delta$ prescription in terms of energies in a specific Lorentz frame, we may easily formulate it in a Lorentz-covariant way: Let $\eta$ be a Lorentz vector with $\eta_{0}>0$ and $\eta^{2} \geq 0$. Then $s_{j}(\sigma)$ is given by

$$
s_{j}(\sigma)=\sum_{a \in\{j\} \cup \pi} \frac{\eta \cdot k_{j}}{\eta \cdot k_{a}} .
$$

Let us look at an example. Figure 1 shows a two-loop eightpoint graph $\Gamma$. There are 35 spanning trees, and each spanning tree defines a cut graph. Figure 2 shows an example of a cut graph corresponding to $\sigma=\{3,9\}$. We also indicate in Fig. 2 an orientation for the edges $e_{3}, e_{5}, e_{6}$, and $e_{9}$. As an example we consider $s_{5}(\sigma)$ and $s_{6}(\sigma)$. The sign of the imaginary part is determined by

$$
\begin{aligned}
& s_{5}(\sigma)=\frac{E_{3}+E_{5}}{E_{3}}, \\
& s_{6}(\sigma)=\frac{E_{3} E_{6}+E_{3} E_{9}+E_{6} E_{9}}{E_{3} E_{9}} .
\end{aligned}
$$

There are ample applications of our result. Let us give three examples. First, our result is directly geared towards numerical methods for higher-order computations $[2,3,6,7,11-33]$ and paves the way to treat two-loop amplitudes numerically in an efficient and automated way. Second, and in a wider context, it sheds new light on the cancellation of infrared singularities. Our result allows us to discuss the singularity structure of loop integrands in terms of on-shell tree diagrams. This will be helpful at next-to-next-to-leading order and beyond [34-63]. Third, and on the formal side, our approach also suggests an extension of the concept of scattering forms [64-67] from tree level towards loops. This will be explored in a future publication.

This work has been supported by the Cluster of Excellence "Precision Physics, Fundamental Interactions, and Structure of Matter" (PRISMA + EXC 2118/1) funded by the German Research Foundation (DFG) within the German Excellence Strategy (Project No. 39083149).

[1] R. P. Feynman, Acta Phys. Pol. 24, 697 (1963).

[2] S. Catani, T. Gleisberg, F. Krauss, G. Rodrigo, and J.-C. Winter, J. High Energy Phys. 09 (2008) 065.

[3] I. Bierenbaum, S. Catani, P. Draggiotis, and G. Rodrigo, J. High Energy Phys. 10 (2010) 073.

[4] C. Baadsgaard, N. E. J. Bjerrum-Bohr, J. L. Bourjaily, S. Caron-Huot, P. H. Damgaard, and B. Feng, Phys. Rev. Lett. 116, 061601 (2016).

[5] C. Bogner and S. Weinzierl, Int. J. Mod. Phys. A 25, 2585 (2010).

[6] S. Becker, C. Reuschle, and S. Weinzierl, J. High Energy Phys. 12 (2010) 013.

[7] S. Becker, C. Reuschle, and S. Weinzierl, J. High Energy Phys. 07 (2012) 090.

[8] T. Kinoshita, J. Math. Phys. (N.Y.) 3, 650 (1962).

[9] T. D. Lee and M. Nauenberg, Phys. Rev. 133, B1549 (1964).

[10] P. Griffiths and J. Harris, Principles of Algebraic Geometry (John Wiley \& Sons, New York, 1994).

[11] I. Bierenbaum, S. Buchta, P. Draggiotis, I. Malamos, and G. Rodrigo, J. High Energy Phys. 03 (2013) 025.

[12] D. E. Soper, Phys. Rev. Lett. 81, 2638 (1998).

[13] D. E. Soper, Phys. Rev. D 62, 014009 (2000).

[14] Z. Nagy and D. E. Soper, J. High Energy Phys. 09 (2003) 055 .

[15] W. Gong, Z. Nagy, and D. E. Soper, Phys. Rev. D 79, 033005 (2009).

[16] S. Buchta, G. Chachamis, P. Draggiotis, I. Malamos, and G. Rodrigo, J. High Energy Phys. 11 (2014) 014.

[17] R. J. Hernandez-Pinto, G. F. R. Sborlini, and G. Rodrigo, J. High Energy Phys. 02 (2016) 044.

[18] S. Buchta, G. Chachamis, P. Draggiotis, and G. Rodrigo, Eur. Phys. J. C 77, 274 (2017).

[19] G. F. R. Sborlini, F. Driencourt-Mangin, R. HernandezPinto, and G. Rodrigo, J. High Energy Phys. 08 (2016) 160.

[20] F. Driencourt-Mangin, G. Rodrigo, and G. F. R. Sborlini, Eur. Phys. J. C 78, 231 (2018).

[21] F. Driencourt-Mangin, G. Rodrigo, G. F. R. Sborlini, and W. J. Torres Bobadilla, J. High Energy Phys. 02 (2019) 143.

[22] M. Assadsolimani, S. Becker, and S. Weinzierl, Phys. Rev. D 81, 094002 (2010).

[23] M. Assadsolimani, S. Becker, C. Reuschle, and S. Weinzierl, Nucl. Phys. B, Proc. Suppl. 205-206, 224 (2010).

[24] S. Becker, D. Götz, C. Reuschle, C. Schwan, and S. Weinzierl, Phys. Rev. Lett. 108, 032005 (2012).

[25] S. Becker and S. Weinzierl, Phys. Rev. D 86, 074009 (2012).

[26] S. Becker and S. Weinzierl, Eur. Phys. J. C 73, 2321 (2013).

[27] D. Götz, C. Reuschle, C. Schwan, and S. Weinzierl, Proc. Sci. LL2014 (2014) 009.

[28] S. Seth and S. Weinzierl, Phys. Rev. D 93, 114031 (2016).

[29] R. Pittau, J. High Energy Phys. 11 (2012) 151.

[30] R. Pittau, Eur. Phys. J. C 74, 2686 (2014).

[31] A. M. Donati and R. Pittau, Eur. Phys. J. C 74, 2864 (2014).

[32] B. Page and R. Pittau, J. High Energy Phys. 11 (2015) 183.

[33] C. Gnendiger et al., Eur. Phys. J. C 77, 471 (2017).

[34] D. A. Kosower, Phys. Rev. D 67, 116003 (2003). 
[35] D. A. Kosower, Phys. Rev. Lett. 91, 061602 (2003).

[36] S. Weinzierl, J. High Energy Phys. 03 (2003) 062.

[37] S. Weinzierl, J. High Energy Phys. 07 (2003) 052.

[38] A. Gehrmann-De Ridder, T. Gehrmann, and E. W. N. Glover, J. High Energy Phys. 09 (2005) 056.

[39] A. Gehrmann-De Ridder, T. Gehrmann, E. W. N. Glover, and G. Heinrich, J. High Energy Phys. 11 (2007) 058.

[40] A. Daleo, A. Gehrmann-De Ridder, T. Gehrmann, and G. Luisoni, J. High Energy Phys. 01 (2010) 118.

[41] R. Boughezal, A. Gehrmann-De Ridder, and M. Ritzmann, J. High Energy Phys. 02 (2011) 098.

[42] T. Gehrmann and P. F. Monni, J. High Energy Phys. 12 (2011) 049.

[43] G. Abelof and A. Gehrmann-De Ridder, J. High Energy Phys. 04 (2011) 063.

[44] G. Abelof, O. Dekkers, and A. Gehrmann-De Ridder, J. High Energy Phys. 12 (2012) 107.

[45] G. Somogyi, Z. Trocsanyi, and V. Del Duca, J. High Energy Phys. 06 (2005) 024.

[46] G. Somogyi, Z. Trocsanyi, and V. Del Duca, J. High Energy Phys. 01 (2007) 070.

[47] G. Somogyi and Z. Trocsanyi, J. High Energy Phys. 01 (2007) 052.

[48] U. Aglietti, V. Del Duca, C. Duhr, G. Somogyi, and Z. Trocsanyi, J. High Energy Phys. 09 (2008) 107.

[49] G. Somogyi and Z. Trocsanyi, J. High Energy Phys. 08 (2008) 042.

[50] G. Somogyi, J. High Energy Phys. 05 (2009) 016.

[51] P. Bolzoni, G. Somogyi, and Z. Trocsanyi, J. High Energy Phys. 01 (2011) 059.
[52] V. Del Duca, C. Duhr, A. Kardos, G. Somogyi, and Z. Trócsányi, Phys. Rev. Lett. 117, 152004 (2016).

[53] G. Somogyi, A. Kardos, Z. Szőr, and Z. Trócsányi, Acta Phys. Pol. B 48, 1195 (2017).

[54] S. Catani and M. Grazzini, Phys. Rev. Lett. 98, 222002 (2007).

[55] S. Catani et al., arXiv:1901.04005.

[56] M. Czakon, Phys. Lett. B 693, 259 (2010).

[57] M. Czakon, Nucl. Phys. B849, 250 (2011).

[58] M. Czakon and D. Heymes, Nucl. Phys. B890, 152 (2014).

[59] J. Gaunt, M. Stahlhofen, F. J. Tackmann, and J. R. Walsh, J. High Energy Phys. 09 (2015) 058.

[60] R. Boughezal, C. Focke, X. Liu, and F. Petriello, Phys. Rev. Lett. 115, 062002 (2015).

[61] R. Boughezal, X. Liu, and F. Petriello, Phys. Rev. D 91, 094035 (2015).

[62] L. Magnea, E. Maina, G. Pelliccioli, C. Signorile-Signorile, P. Torrielli, and S. Uccirati, J. High Energy Phys. 12 (2018) 107.

[63] L. Magnea, E. Maina, G. Pelliccioli, C. Signorile-Signorile, P. Torrielli, and S. Uccirati, J. High Energy Phys. 12 (2018) 062.

[64] N. Arkani-Hamed, Y. Bai, and T. Lam, J. High Energy Phys. 11 (2017) 039.

[65] S. Mizera, Phys. Rev. Lett. 120, 141602 (2018).

[66] L. de la Cruz, A. Kniss, and S. Weinzierl, J. High Energy Phys. 03 (2018) 064.

[67] N. Arkani-Hamed, Y. Bai, S. He, and G. Yan, J. High Energy Phys. 05 (2018) 096. 